\title{
ATENÇÃO FARMACEUTICA AOS IDOSOS HIPERTENSOS: UM ESTUDO DE CASO DO MUNICÍPIO DE APERIBÉ, RJ
}

\author{
Jessica Christiny MILLER, Nikyallan Soares RODRIGUES, Natalha Felisbino RIBEIRO, Juliano Gomes \\ BARRETO* \& Cristiano Guilherme Alves de OLIVEIRA
}

Universidade Iguaçu - Campus V, Itaperuna, Rio de Janeiro, Brasil.

*Autor para correspondência: julianobarreto@ hotmail.com

http://dx.doi.org/10.18571/acbm.093

\section{RESUMO}

A hipertensão arterial constitui-se como uma patologia muita das vezes assintomática, silenciosa e identificável por meio da aferição da pressão arterial, podendo apresentar valores persistentes maiores ou iguais a $140 \mathrm{mmHg}$ para pressão sistólica e $90 \mathrm{mmHg}$ para diastólica. Os idosos são os que mais sofrem com esta doença. Assim, a função do farmacêutico é sempre orientar sobre os medicamentos a serem administrados pelos idosos já que estes possuem pouco esclarecimento e compreensão a respeito das maneiras de sua utilização. Com esta pesquisa, buscou-se verificar como é realizada a atenção farmacêutica aos idosos hipertensos numa farmácia do Município de Aperibé, RJ, bem como a análise do uso indevido de medicamentos pelos idosos, o papel que o profissional desempenha neste processo e as formas possíveis de prevenir o aumento da pressão arterial pela redução dos fatores de risco. Este trabalho possui como metodologia de pesquisa um estudo qualiquantitativo com procedimentos metodológicos pautados na revisão bibliográfica, utilizando como forma de avaliação a aplicação de questionário estruturado. Foram entrevistados 100 idosos com hipertensão arterial: destes, 13\% receberam o diagnóstico de hipertensão arterial antes dos 40 anos de idade, 29\% foram diagnosticados entre os 41 e 50 e, $58 \%$ foram após 50 anos. Constata-se que no município estudado a maioria dos idosos obteve conhecimento da patologia após os 50 anos.

Palavras-chave: Hipertensão Arterial; Idosos; Atenção Farmacêutica; prevenção.

\begin{abstract}
Arterial hypertension establishes as an almost asymptomatic pathology, silent and identifiable through as blood pressure measurement and may have persisted values greater than or equal to $140 \mathrm{mmHg}$ for systolic pressure and $90 \mathrm{mmHg}$ for diastolic. The elderly are the most affected by this disease. Therefore, the role of the pharmacist is always advise on the drugs to be administered by the elderly since they have little explanation and understanding about the ways of their use. With this research, it sought to check as it is held pharmaceutical care to the elderly hypertensive patients on a pharmacy in Aperibé town in the state of Rio de Janeiro as well as the analysis of the misuse of medicines by the elderly, the role of the professional in this process and the possible ways to prevent high blood pressure by reducing risk factors. This work has as research methodology a qualitative and quantitative study with methodological procedures guided by literature review, using as a means of assessing and implementing a structured questionnaire. There were interviewed 100 elderly people with high blood pressure: of these, $13 \%$ were diagnosed with hypertension before 40 years old, $29 \%$ were diagnosed between 41 and 50 , and $58 \%$ were after 50 years. It confirms that the municipality studied the most seniors got pathology knowledge after 50 years.
\end{abstract}

Keywords: Arterial Hypertension; Elderly; Pharmaceutical Care; Prevention. 


\section{Introdução}

A hipertensão arterial é uma patologia que, maioria dos casos, não exibe sintomas. Seu diagnóstico em quase sua totalidade se dá por uma aferição da pressão arterial de maneira pontual. O diagnóstico de hipertensão arterial é firmado quando valores persistentes maiores ou iguais a $140 \mathrm{mmHg}$ para pressão sistólica e $90 \mathrm{mmHg}$ para diastólica são identificados (CARLOS et al., 2008).

O controle da pressão arterial nos pacientes hipertensos se inicia a partir de uma prescrição correta, passando pela aceitação do paciente ao tratamento proposto, bem como seu controle programado com aferições esporádicas, retorno às consultas de reavaliação e mudança no estilo de vida. O primeiro passo para se obter sucesso no tratamento é a aceitação da patologia. Posteriormente, o hipertenso deve buscar o conhecimento sobre as possíveis complicações e realizar a adesão ao tratamento (SILVA, 2010).

A hipertensão arterial em $95 \%$ dos casos não possui uma causa identificável, tendo o paciente o diagnostico de hipertensão arterial primária ou essencial. Nestes casos, o indivíduo terá que fazer o controle pressórico por toda a vida. Os idosos são os que mais são diagnosticados com hipertensão arterial. Com isso, a atenção farmacêutica ao paciente cresce cada vez mais. Adoecimento e morte de idosos são consequências de vários fatores, sendo um grande desafio para as políticas publicas e de saúde (CINTRA et al., 2011).

Além do tratamento farmacológico, outras formas para realizar o controle da pressão arterial são utilizadas como o abandono do tabaco, a redução do índice de massa corpórea e gordura visceral, diminuição no consumo de bebidas alcoólicas, controle da ingesta de sal (sódio) e exercícios físicos. Alguns autores questionam a eficácia das intervenções educacionais na mudança do estilo de vida dos idosos e, consequentemente, a prevalência dos fatores de riscos nas doenças crônicas (ACHUTTI, 1997).

Em uma parcela substancial dos pacientes hipertensos, somente a mudança no estilo de vida não é suficiente para o correto controle da pressão arterial. Assim, o tratamento farmacológico torna-se fundamental. Ele vai depender não somente dos níveis de pressão arterial, mas também da coexistência de fatores de risco e de lesões em outros órgãos. A hipertensão arterial afeta mais intensamente os homens. Essa situação se inverte quando as mulheres chegam à menopausa e terceira idade, onde nelas passa a ter maior incidência, aumentando, assim, a sua prevalência (ACHUTTI, 1997).

Diante da automedicação e da utilização inadvertida dos anti-hipertensivos por idosos, busca-se discutir o papel dos profissionais de saúde, principalmente o farmacêutico de modo que seja possível controlar e prevenir os fatores de riscos nos idosos.

Este trabalho tem como objetivo verificar como é realizada a atenção farmacêutica aos idosos hipertensos em uma farmácia no município de Aperibé, Estado do Rio de Janeiro. Sua relevância se justifica por não existir trabalhos relacionados ao tema no município estudado, abordando a população idosa que necessita de informações sobre a utilização das medicações.

\section{Revisão da literatura}

\subsection{Atuação do farmacêutico}

O efeito do tratamento pode estar relacionado ao aparecimento de reações adversas causadas pelo medicamento. $\mathrm{O}$ efeito indesejado deste medicamento pode ser uma das causas para promover a interrupção do tratamento. Nesta reação a orientação farmacêutica ao paciente idoso facilitaria a volta ao tratamento alertando-o das reações adversas causadas ao paciente e uma possível substituição do medicamento (ROCHA et al., 2008). 
O profissional farmacêutico deve sempre orientar e educar o paciente idoso sobre sua patologia e os medicamentos a serem administrados, o foco da atenção farmacêutica é o bem estar e a qualidade de vida do paciente idoso. O uso de vários medicamentos pode ser prejudicial à saúde do paciente, a maioria dos idosos para de administrar os seus medicamentos quando se sentem melhor e acabam aumentando as doses dos medicamentos quando volta a sentir novamente os sintomas (ROCHA et al., 2008).

Assim como Rocha et al. (2008) ressaltaram, um papel muito importante do farmacêutico é anotar os horários dos medicamentos para o paciente e a quantidade de cada medicamento para ser conferida na próxima visita domiciliar.

Em outros países o farmacêutico que é o profissional responsável adequado para orientar sobre os medicamentos aos idosos, quando o farmacêutico tem contato frequente com o paciente ele pode sempre informar ao paciente a natureza da doença crônica e identificar as razões do tratamento. A participação do farmacêutico no controle da hipertensão arterial consiste na seleção dos medicamentos, gerenciamento do estoque do armazenamento correto, mas, principalmente na promoção da atenção farmacêutica ao paciente (LIRA JÚNIOR et al, 2006).

\subsection{Medicamentos utilizados no tratamento da hipertensão arterial}

Os idosos hipertensos devem fazer o tratamento com o objetivo da redução da pressão arterial (PA), para níveis inferiores a 140/90 mmHg. Devem ser evitados os níveis de PA diastólica menores que $65 \mathrm{mmHg}$. Em pacientes com níveis de pressão arterial muito elevado valores da pressão arterial sistólica até $160 \mathrm{mmHg}$ podem ser considerados inicialmente tolerados. A maioria dos pacientes necessita de dois ou mais classes de fármacos diferentes para o controle ideal da pressão arterial (PERROTTI et al., 2002).

Existem vários medicamentos muito bons para o tratamento da hipertensão arterial, cada um com sua classe específica. Tem-se o Losartana que é da classe dos Antagonistas da Angiotensina II, são os que representam menor risco de efeitos colaterais entre as classes do antihipertensivo. O Losartana é utilizado para hipertensão arterial e na insuficiência cardíaca, seus efeitos são semelhantes aos inibidores da enzima conversora da angiotensina (ECA), são usados por via oral os seus efeitos adversos são raros, um deles são hepatotoxidade e edema ele também é contra indicado pra gestante (MIRANDA et al., 2002).

O Hidroclorotiazida é da classe dos diuréticos tiazídico, ele é essencial para o tratamento de hipertensão arterial, tem vários efeitos colaterais como hipocalemia e hiperuricemia (PUCCI et al., 2012).

O Atenolol e o Propanolol são da classe dos $\beta$-Bloqueadores, todos os dois são usados para hipertensão arterial, mas o Atenolol é o que tem menor efeito no sistema nervoso central, que tem como efeito colateral depressão, sonolência, confusão, distúrbio do sono. Já o propanolol os seus efeitos colaterais mais frequentes são dor de cabeça, humor depressivo, cansaço (MIRANDA et al., 2002).

O Captopril é também indicado para pacientes hipertensos, da classe da ECA. Os efeitos colaterais são tosse seca, alteração do paladar, em gestante é contraindicado, porque pode causar má formação do feto. Na pesquisa de Schroeter, 38,4\% usavam os medicamentos da classe da ECA, 26\% usavam $\beta$-bloqueadores e 59,4\% utilizavam diuréticos (SCHROETER et al., 2007).

O Nifedipino pertence à classe dos bloqueadores dos canais de cálcio, é indicado para pacientes que fazem tratamento de hipertensão arterial, tem vários efeitos colaterais, porem os mais comuns são rubor facial, tonteira, cefaleia e edema periférico. O Nifedipino tem que ser usado controladamente, pois se usar em altas doses pode causar depressão e parada cardíaca (KOHLMANN JUNIOR et al., 1999). 
2.3 Sinais e sintomas apresentados na utilização inadequada dos medicamentos antihipertensivos pelos idosos

A terapia farmacológica anti-hipertensiva no paciente idoso deve ser usada com muito cuidado, pois se tem que escolher o medicamento apropriado para cada paciente, escolher sempre os que têm menos efeitos colaterais, os que reduzem a morbidade e mortalidade cardiovascular são sempre os melhores medicamentos (SCHROETER et al., 2007).

Hipertensão arterial é uma doença com sinais e sintomas obscuros e muito das vezes ausentes, com isso as informações dos profissionais de saúde é fundamental, explicando o paciente sempre como é a gravidade da doença e os benefícios do tratamento (JARDIM et al., 2006).

Os adultos com mais de 65 anos de idade consomem entre $30 \%$ à $40 \%$ de todas as medicações prescritas. $\mathrm{O}$ organismo do idoso vai sofrendo alterações em praticamente todos os órgãos e sistemas e com isso os idosos tornam-se mais frágil aos efeitos colaterais da medicação (BALLONE, 2002).

Os anti-hipertensivos devem ser utilizados isoladamente ou em combinação com outros medicamentos, podem reduzir os batimentos cardíacos, agravar a depressão, causar impotência, sedação e tontura quando a pessoa levanta (GOODMAN, 1996).

Os efeitos mais comuns referidos por Asperheim (1994), como consequência do uso de fármacos anti-hipertensivos são: tonteira, hipotensão ortostática, constipação, bradicardia, ginecomastia (com o uso de Metildopa), sonolência e impotência (com o uso de Verapamil).

Os sinais e sintomas do Nifedipino representado pela classe dos antagonistas de cálcio são vertigens, constipação intestinal, edema perimaleolar e queda excessiva da pressão arterial (OLIVEIRA et al., 1999).

\subsection{Formas de combater os efeitos indesejáveis dos medicamentos}

Uma das formas muito importante em combater os efeitos indesejáveis dos medicamentos é o tratamento não medicamentoso da hipertensão, são medidas não medicamentosas como a redução do peso que ajuda muito no tratamento do paciente hipertenso, consumir sempre frutas e vegetais, interrupção do tabagismo, diminuição do consumo de bebidas alcoólicas, reduzir o consumo exagerado de sal e fazer sempre exercícios físicos que resultam em queda significativa da pressão arterial, a combinação dessas medidas pode resultar em efeitos favoráveis para o controle da pressão arterial, por tanto essas medidas devem ser sempre aconselhada para pacientes que fazem tratamento de hipertensão arterial principalmente em pessoas idosas (PEDROSA, DRAGER, 2008).

O Propanolol e o Atenolol agente $\beta$-bloqueador atuam sobre o metabolismo da glicose como hipoglicemiante (são medicamentos que provocam uma diminuição da glicose plasmática). Os anti-hipertensivos não devem ser administrados com alimentos como banana, goiaba e maçã, pois retardará ou impedirá o sua absorção (BRANCO, 1997).

\section{Materiais e Métodos}

A metodologia aplicada nesta pesquisa foi pautada em um estudo qualiquantitativo de ordem bibliográfica com fundamentação teórica em publicações e artigos científicos e relacionados ao trabalho que o profissional farmacêutico desempenha diante de idosos com hipertensão arterial no município de Aperibé, RJ. Foi utilizada como forma de avaliação a aplicação de questionário estruturado com perguntas claras, formuladas de acordo com os resultados que se pretende alcançar. Os questionários foram aplicados em uma Drogaria 
localizada no município de Aperibé, que se situa ao Noroeste do Estado do Rio de Janeiro, tendo segundo IBGE (2014), população estimada de 10.882 habitantes.

Com estas avaliações do estudo realizado, tornará possível traçar o perfil dos usuários de medicamentos anti-hipertensivos atendidos na drogaria, a idade mais frequente dos portadores de hipertensão arterial, e os principais fatores envolvidos na pré-disposição de sua ocorrência.

\section{Resultados e Discussão}

A hipertensão arterial responde por grande parte da morbimortalidade da população brasileira, sendo fator preponderante de acometimento de doenças isquêmicas e causa mortis. Desta forma, a evidência de trabalhos que tragam a luz os fatores que identifiquem as nuances desta patologia se faz necessário sobre o papel do farmacêutico e demais profissionais no âmbito da multidisciplinaridade em pacientes da terceira idade.

Foram estudados 100 pacientes idosos com hipertensão arterial. Destes, 13\% receberam o diagnóstico de hipertensão arterial antes da quinta década de vida, 29\% foram diagnosticados na quinta década e, $58 \%$ foram após a sexta década. Constata-se que, no município estudado, a maioria dos idosos obteve conhecimento da patologia após os 50 anos como observado na figura 1.

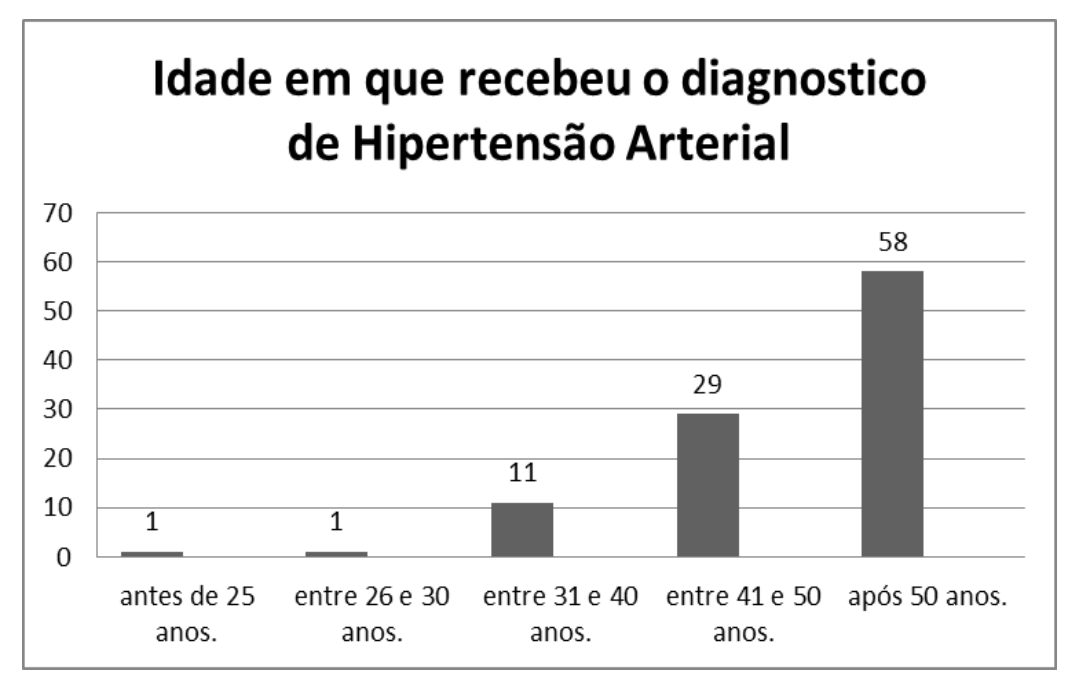

Figura 1: Idade dos idosos em que receberam diagnóstico de hipertensão arterial.

Em um estudo realizado por Ramos \& Maia (2013) em uma Unidade Saúde da Família, no município de Patos, Estado da Paraíba constatou-se que em relação à faixa etária $36 \%$ esta entre 71 a 75 anos, $30 \%$ esta na faixa de 66 a 70,17\% entre 60 a 65 anos, $10 \%$ possui mais de 80 anos e 76 a 80 anos tem apenas $7 \%$. O que denota uma prevalência da patologia em idades mais avançadas e a necessidade de um diagnóstico ou acompanhamento dos idosos em relação aos valores pressóricos e comorbidades associadas.

Com relações as informações prestadas por profissionais de saúde sobre a utilização correta de medicamentos, pode se observar na Figura 2 que $25 \%$ dos entrevistados afirmaram receber esclarecimentos pelo farmacêutico, enquanto os outros $75 \%$ afirmam receber através do médico. Como evidencia, pode-se demonstrar a necessidade de um trabalho mais eficaz em relação aos serviços farmacêuticos, no tocante ao plano de cuidados e adesão no processo farmacológico.

Sendo, o farmacêutico clínico como elo entre o diagnóstico médico e o sucesso terapêutico. Para tal, faz necessária a maior presença do profissional como membro ativo da cadeia de assistência farmacêutica incorporando seus conhecimentos práticos e teóricos no 
decorrer do tratamento. Ainda, sim se destaca rotineira e permanente desatenção nos cuidados com os pacientes idosos em relação às informações sobre os medicamentos, já que esse insumo se trata em especial delegação única e privativa do profissional farmacêutico. Não só sua dispensação, mas a zelosa pratica de estipular parâmetros e condutas que tragam aos usuários melhoras significativa no objetivo tratado pelo ato terapêutico.

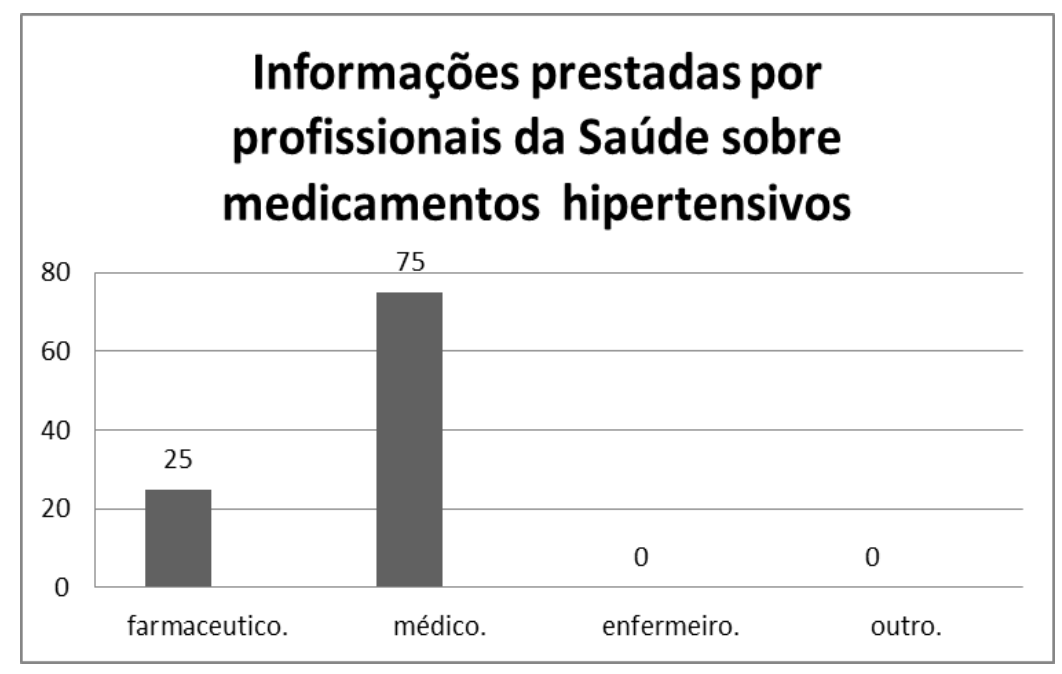

Figura 2: Informações prestadas por profissionais da saúde para os idosos sobre medicamentos.

A figura 3 a seguir demonstra que $75 \%$ dos entrevistados afirmaram não utilizar nenhum tipo de drogas lícitas ou ilícitas, $12 \%$ atribuíram o uso de álcool e $17 \%$ de cigarros. De acordo com estudo realizado por Monteiro (2005), demonstra-se que entre as pessoas entrevistadas 3,65\% fazem utilização de bebidas alcoólicas e 8,54\% eram tabagistas. Péres et al. (2003), informa que apenas $20 \%$ não são tabagistas.

Apesar de não tão discrepantes os dados obtidos nesse estudo se amplificam, uma vez que a população estudada apresenta uma longevidade, o que traria possivelmente anos de exposição ao tabaco o que não foi observado pelo mesmo trabalho. Destaca-se que em pacientes com mais de 65 anos o risco cardíaco aumenta consideravelmente quando os mesmos apresentam o tabagismo como comorbidade. Sendo, papel fundamental para a prática farmacêutica as orientações necessárias para as mudanças do estilo de vida (MEV) com recomendações e controle no habito de beber e modificações no perfil de fumante dos idosos.

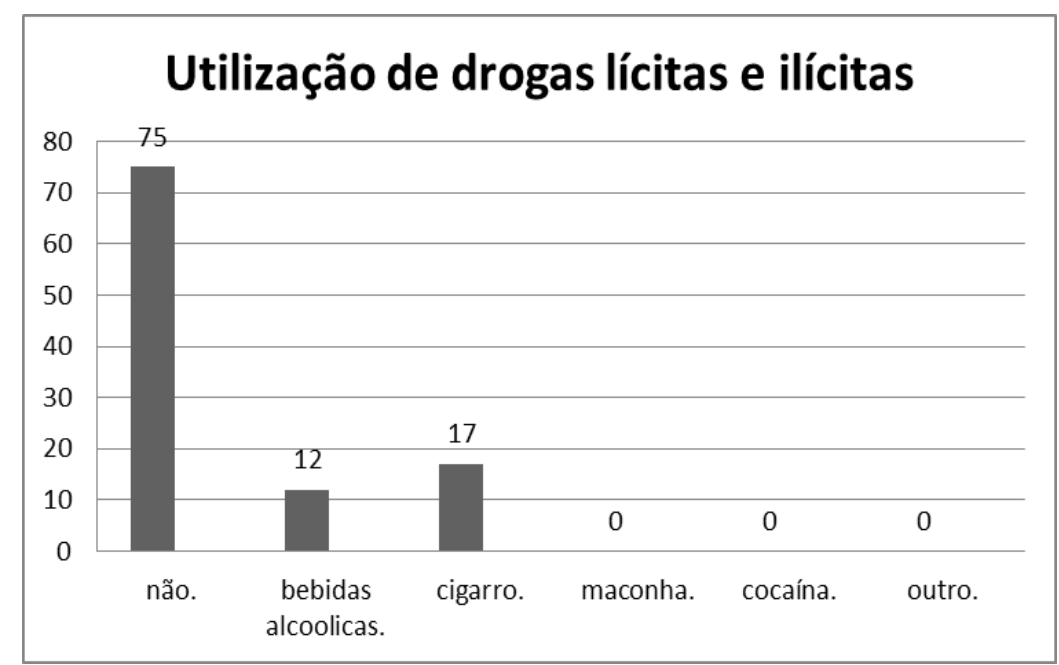

Figura 3: Idosos que fazem uso de drogas lícitas ou ilícitas. 
Os medicamentos citados pelos idosos foram predominantemente Losartana (56\%), Captopril (38\%), Atenolol (23\%), Nifedipino, Hidroclorotiazida e propanolol tiveram 5\%. Segundo Cavalcante 2014, os anti-hipertensivos mais utilizados foram Hidroclorotiazida (27\%), Captopril (22\%) e Losartana (15\%) conforme apresentado na figura 4.

Pode-se descrever que o perfil farmacológico adotado tem como referencial os fármacos adotados pela Relação Nacional de Medicamentos Essenciais (RENAME) e distribuídos pelo sistema Farmácia Popular (FP) programa assistencial de distribuição de medicamentos pelo governo com custo zero ao usuário de qualquer origem, sendo publico ou privado acompanhado de receituário, documento com foto e CPF. Dentre os listados temos: losartana potassica de 50 $\mathrm{mg}$, captopril de $25 \mathrm{mg}$, propranolol de $40 \mathrm{mg}$, atenolol de $25 \mathrm{mg}$, hidroclorotiazida de $25 \mathrm{mg}$. Sendo que o medicamento nifedipino disponível somente na rede publica por intermédio SUS.

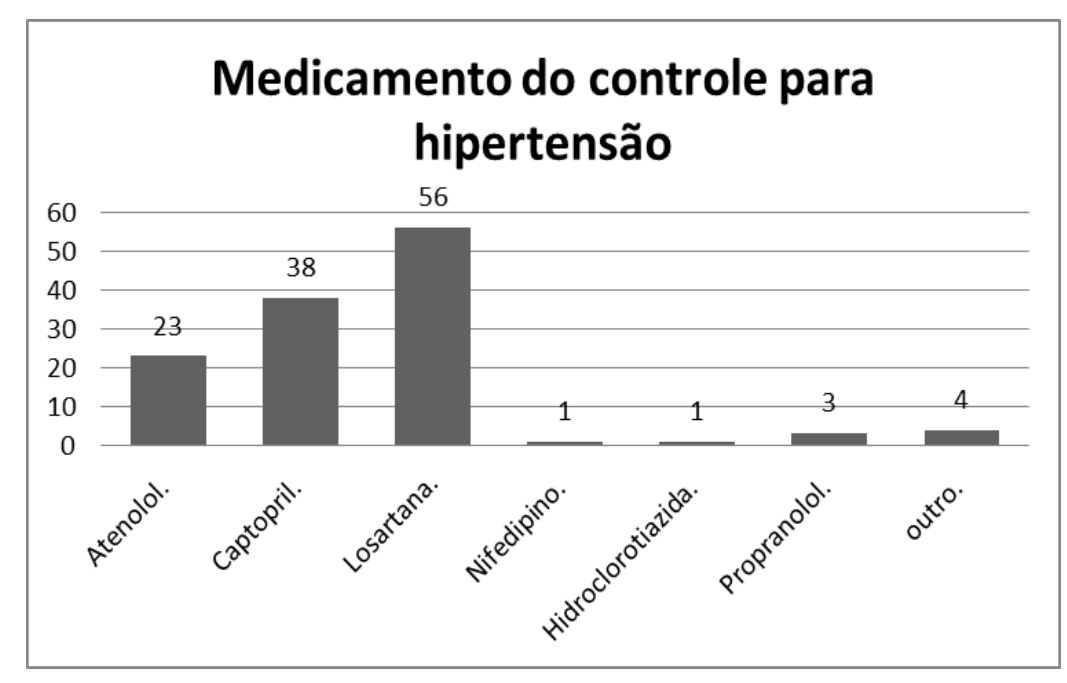

Figura 4: Medicamentos utilizados no controle de hipertensão arterial.

Os fármacos hipertensivos, em destaque, os antagonistas dos receptores da angiotensina (ARAs) como a losartana tem ganhado notório desempenho por apresentar dentre os acima citados pertencentes à FP resultados mais eficientes e menores taxas de insegurança.

$\mathrm{Na}$ figura 5 pode se destacar que $66 \%$ dos idosos relataram não fazer uso de outros medicamentos em associação aos anti-hipertensivos, enquanto 34\% afirmam utilizar outros medicamentos de forma concomitante.

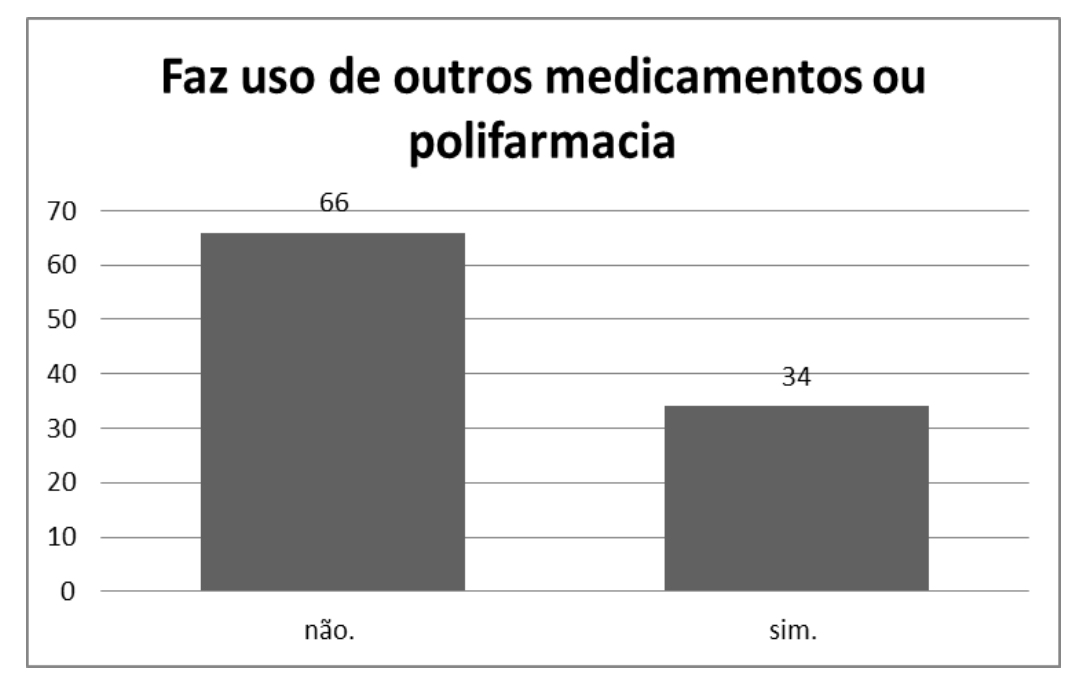

Figura 5: Números de idosos que fazem uso de outro tipo de patologia. 
Com relação ao histórico genético/familiar de hipertensão arterial, $62 \%$ dos entrevistados disseram que apresentavam casos em parentes de primeiro grau e $41 \%$ não tem histórico familiar como observado na figura 6 a seguir.

Segundo Romero et al. (2010), 50,8\% das pessoas entrevistadas afirmaram ter portadores de hipertensão arterial na família, sendo que o maior numero foi de irmãos com $62,1 \%$.

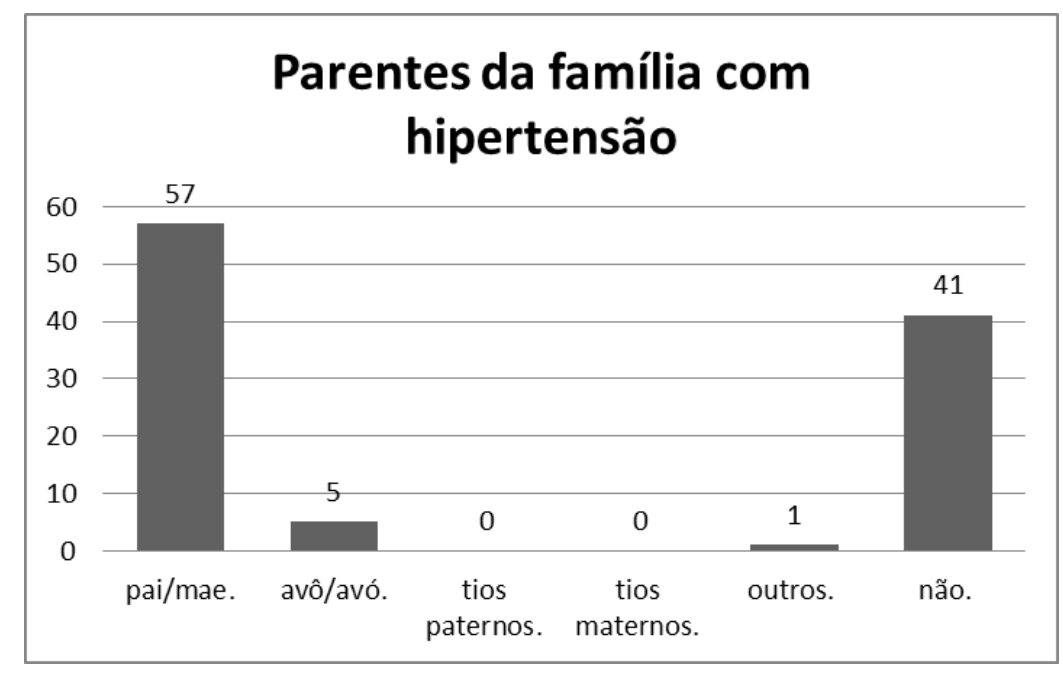

Figura 6: Idosos que possuem casos de hipertensão arterial na família.

Na figura 7 a seguir, pode se demonstrar que $98 \%$ dos entrevistados relataram fazer dieta alimentar evitando o consumo de alimentos ricos em sódio, gorduras ou carboidratos/açúcares, enquanto apenas $2 \%$ não tem nenhum tipo de restrição alimentar.

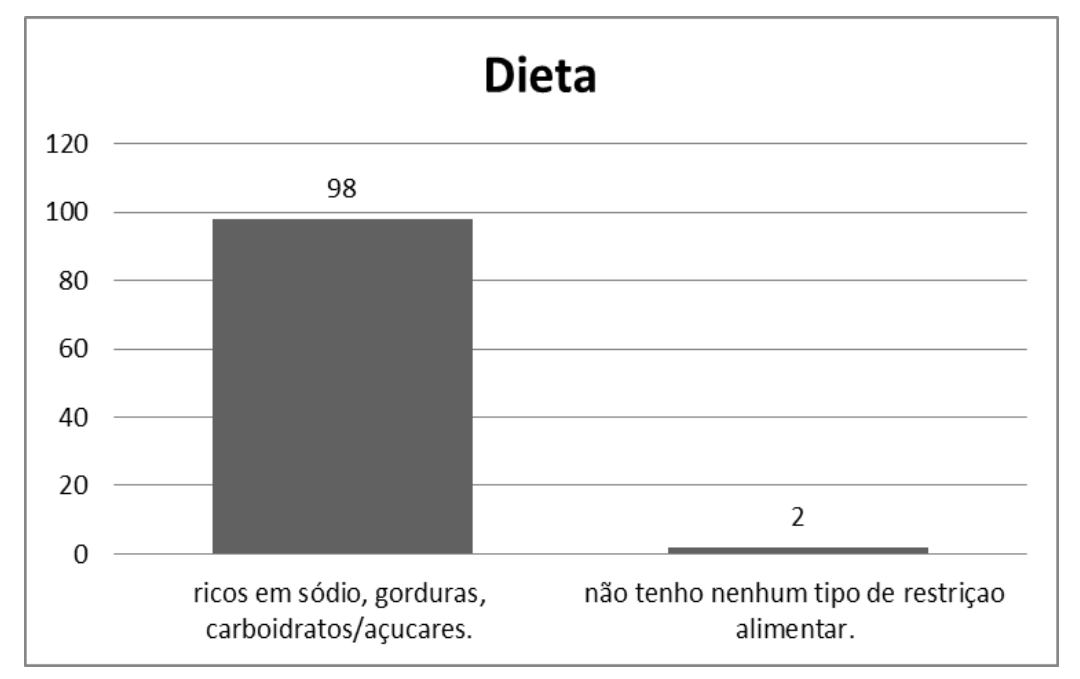

Figura 7: Idosos que fazem dieta evitando o consumo descontrolado de sódio, gorduras e carboidratos/açucares.

Tal fato, representado nas figuras acima, predisponente com MEV torna se necessário a intervenção profissional no acompanhamento e definição de parâmetros para a melhoria na qualidade de vida dos idosos. Ainda cabe salientar a importância na aferição de medidas como índice de massa corporal (IMC), valores de lipídios com observância em possíveis dislipidemias 
praticam de exercícios físicos e demais situações que possam agravar o risco de doenças pressóricas e comprometimento com risco de sequelas e óbito.

\section{Considerações Finais}

O resultado desse estudo demonstra que o diagnóstico de hipertensão arterial pode ser aventado em diversas fases da vida. $\mathrm{O}$ indivíduo pode se tornar hipertenso em qualquer idade, porém as maiores vítimas neste estudo foram aquelas com mais de 60 anos. Muitas delas afirmaram ter algum histórico familiar de hipertensão, predisposição genética, mostraram que fazem sempre dieta e procuram não abusar dos alimentos que contém muito sódio e carboidratos.

O fármaco mais utilizado pelos pacientes avaliados nesta pesquisa foi a Losartana. A maioria dos pacientes avaliados recebeu o diagnóstico e a orientação sobre sua patologia pelos médicos. Foi observado que eles buscam melhorias nas condições de saúde e qualidade de vida, com o tratamento adequado e utilização de medicamentos corretos para controle da pressão arterial.

\section{Referências}

ACHUTTI, A. C.; ACHUTTI, V. A. R. Aspectos Epidemiológicos. In: AMODEU, C.; LIMA, E. G, VARQUEZ, E. C. Hipertensão arterial. São Paulo (SP): Sarvier; 1997. p.11-22.

ASPERHEIM, M. K. Farmacologia para Enfermagem. 7. ed. Rio de Janeiro: Guanabara Koogan, 1994.

BALlOne, J. G. Uso de Medicamentos por Idosos e Iatrogenia. Disponível em: <http: sites.uol.com.br/gballone/geriat/medicam. html >. Acesso em: 16 out. 2015.

BRANCO, C. C. C. Interaçao Farmaco Nutrientes. Revista RECCS. Fortaleza. N9, P.30-34. 1997.

CARLOS, P. R.; PALHA, P.F.; VEIGA,E. V.; BECCARIA, L.M. Perfil de Hipertensos em um Núcleo de Saúde da Família. Arq Ciênc Saúde. 2008 out/dez;15(4):176-81.

CAVALCANTE, N. C. Potenciais Interações Medicamentosas Na Farmacoterapia De Hipertensos Cadastrados No Programa Hiperdia De Uma Unidade Integrada De Saúde Da Família Do Município De João Pessoa-Pb. 2014. Universidade Federal Da Paraíba Centro De Ciências Da Saúde Departamento De Ciências Farmacêuticas. 2014.

GOODMAN, L. S. As Bases Farmacológicas da Terapêutica. Rio de Janeiro: McGriw-Hill, 1996.

JARDIM, P. C. B. V.; JARDIM, T. S. V. Modelos de Estudos de Adesão ao Tratamento AntiHipertensivo. Rev Bras Hipertens. vol.13(1): 26-29, 2006.

KOHLMANN JUNIOR, O; GUIMARÃES, A. C.; CARVALHO, M. H. C.; JUNIOR, H. C. C.; MACHADO, C. A.; PRAXEDES, J. N.; SANTELLO, J. L.; NOBRE, F.; JUNIOR, D. C. M. III Consenso Brasileiro de Hipertensão Arterial. Arq Bras Endocrinol Metab vol.43 no. 4 São Paulo Aug. 1999.

LIRA JÚNIOR, D. P; AMARAL, R. T.; VEIGA, E. V.; CÁRNIO, E. C.; NOGUEIRA, M. S.; PELÁ, I. R. A Farmacoterapia No Idoso: Revisão Sobre A Abordagem Multiprofissional No 
Controle Da Hipertensão Arterial Sistêmica. Rev Latino-Am Enfermagem. 2006 maio-junho; 14(3):435-41.

MIRANDA, R. D., PERROTI, T. C., BEllinAZZI, V. R., NOBREGA, T. M., CENDOROGLO, M. S., NETO, J. T. Hipertensão Arterial no Idoso: Peculiaridades na Fisiopatologia, no Diagnóstico e no Tratamento. Rev Bras Hipertens 9: 293-300, 2002.

MONTEIRO, P. C.; SANTOS, F. S.; FORNAZARI, P. A.; CESARINO, C. B. Características Biossociais, Hábitos de Vida e Controle da Pressão Arterial dos Pacientes em um Programa de Hipertensão. Arq Ciênc Saúde. 2005 abr-jun;12(2):73-9

PEDROSA, R.P.; DRAGER. D. L. Hipertensão arterial sistêmica. RBM Rev Bras Med. 2008; 65(12): 74-84.

PÉRES, D. S.; MAGNA, J. M.; VIANA, L. A. Portador De Hipertensão Arterial: Atitudes, Crenças, Percepções, Pensamentos E Práticas. Revista Saúde Publica. 2003;37(5):635-42.

PERrotTi, T. C., Filho, J. C., UEHARA, C. A., Filho, C. M. A., MIRANDA, R. D. Tratamento Farmacológico da Hipertensão no Idoso. Rev Bras Hipertens vol.14(1): 37-41, 2007.

PUCCI, N.; PEREIRA, M. R.; VINHOLES, D. B.; PUCCI, P.; CAMPOS, N. D. Conhecimento Sobre Hipertensão Arterial Sistêmica e Adesão ao Tratamento Anti-Hipertensivo em Idosos. Rev Bras Cardiol. 25(4):322-329, julho/agosto, 2012.

RAMOS, F.F.A; MAIA, J.A. Tratamento da hipertensão arterial entre usuários idosos assistidos pela enfermagem numa unidade básica de saúde da família. REBES, v.3, n.2, p.9-16, abr.-jun. 2013.

ROCHA, C. H.; OLIVEIRA, A. P. S.; FERREIRA, C.; FAGGIANI, F. T.; SCHROETER, G.; SOUZA, A. C. A.; DECARLI. G. A.; MORRONE, F. B.; WERLANG, M. C. Adesão à Prescrição Médica em Idosos de Porto Alegre, RS. Ciência \& Saúde Coletiva, 13(Sup): 703710, 2008.

ROMERO, A. D.; SILVA, M. J.; SILVA, A. R. V.; FREITAS, R. W. J. F.; DAMASCENO, M. M. C. Características De Uma População De Idosos Hipertensos Atendida Numa Unidade De Saúde Da Família. Rev. Rene. Fortaleza, v. 11, n. 2, p. 72-78, abr./jun.2010

OLIVEIRA, J. J.; SILVA, S. R. A. S. O idoso com Hipertensão Arterial. Rev. Bras. Med., Rio de Janeiro, v. 56, n. 7, p. 566-578, 1999.

SCHROETER, G.; TROMBETTA, T.; FAGGIANI, F. T.; GOULART, P. V.; CREUTZBERG, M.; VIEGAS, K.; SOUZA, A. C. A.; CARLI, G. A. MORRONE, F.B. Terapia AntiHipertensiva Utilizada por Pacientes Idosos de Porto Alegre/RS, Brasil. Scientia Medica, Porto Alegre, v. 17, n. 1, p. 14-19, jan./mar. 2007.

SILVA, M. E. D. C. Representações sociais da hipertensão arterial elaboradas por portadoras e profissionais da saúde: uma contribuição para a enfermagem. 2010. $153 \mathrm{f}$. Trabalho de Conclusão de Curso (especialização) - Universidade Federal do Piauí, Teresina. 